\title{
Study of the effect of miR-124 and the SOX9 target gene in Hirschsprung's disease
}

\author{
JIE MI, DONG CHEN, MEI WU, WEILIN WANG and HONG GAO \\ Laboratory of Congenital Malformation, Ministry of Public Health, Shengjing Hospital of China Medical University, \\ Shenyang, Liaoning 110004, P.R. China
}

Received July 11, 2013; Accepted February 25, 2014

DOI: $10.3892 / \mathrm{mmr} .2014 .2022$

\begin{abstract}
Hirschsprung's disease (HSCR) is a polygenic disease, of which the cause remains to be elucidated. It has been suggested that SRY-related HMG-box 9 (SOX9) is fundamental for the correct development of oligodendrocytes and astrocytes; however, not the development of neurons. There are currently no reports regarding SOX 9 expression in patients with HSCR; therefore, the present study aimed to investigate the expression of microRNA-124 (miR-124) and its target gene, SOX9, in HSCR. Quantitative polymerase chain reaction (qPCR), western blot analysis and immunohistochemistry were used to detect the mRNA and protein expression of miR-124 and SOX9 in patients with HSCR. miR-124 expression was observed to be markedly higher in stenotic colon segment tissues compared with normal colon segment tissues in patients with HSCR. Furthermore, mRNA and protein analyses revealed that SOX9 expression was also higher in the stenotic colon segment tissues compared with the normal colon segment tissues. In conclusion, these data suggest that miR-124 and its target gene, SOX9, are overexpressed in the stenotic colon segment of patients with HSCR, and may have a significant role in the development of HSCR.
\end{abstract}

\section{Introduction}

Hirschsprung's disease (HSCR) is characterized by aganglionosis; a loss of enteric ganglia in the distal section of the gut $(1,2)$. HSCR represents the predominant genetic cause of functional intestinal obstruction with an average incidence of 20/100,000 live births worldwide. There is a considerable ethnic disparity in the incidence of the disease, with an incidence of 28/100,000 live births in individuals of Asian

Correspondence to: Professor Hong Gao, Laboratory of Congenital Malformation, Ministry of Public Health, Shengjing Hospital of China Medical University, 36 Sanhao Street, Heping District, Shenyang, Liaoning 110004, P.R. China

E-mail: gaohong515@sina.com

Key words: Hirschsprung's disease, miR-124, target gene SOX9, expression descent (3). Aganglionosis is a disorder of the enteric nervous system (ENS) in which ganglion cells fail to innervate the lower gastrointestinal tract during embryonic development (4).

As a neurocristopathy, HSCR is a complex disease influenced by multiple genetic and environmental factors. Mutations in genes with significant roles in the formation of the ENS have been identified in patients with HSCR, including rearranged during transfection (RET) (5-7), endothelin receptor type B (EDNRB) (8-10), endothelin-3 (EDN3) $(11,12)$, glial cell line-derived neurotrophic factor (GDNF) (13-15) and SRY-related HMG-box (SOX) $10(16,17)$. SOX10 is a major gene associated with HSCR. In the human colon, SOX10 expression is restricted to the ENS, and is present in neuronal and glial cells. The expression of SOX10 was observed to be consistently lower in the aganglionic segments of the colon than in the hypoganglionic and normal segments (18).

SOX9 is a member of the SOXE group of genes along with SOX8 and SOX10, and is markedly expressed, initially in neural stem cells and later in glial cells, in the developing central nervous system (CNS). It has been identified that SOX9 is crucial for the correct development of oligodendrocytes and astrocytes, but not neurons (19). Moreover, it has been shown that SOX9 is present in a small population of subventricular zone (SVZ) cells in the postnatal and adult mouse brain (20). However, there are no reports regarding SOX9 expression in patients with HSCR.

MicroRNAs (miRNAs) are a class of short, noncoding RNA genes that have been reported to demonstrate essential roles in cell growth and apoptosis, hematopoietic lineage differentiation and tumorigenesis $(21,22)$. The miR-124 family of miRNAs are a key focus of research into miRNA function in humans and animals. It has been reported that miR-124 is a significant regulator of the temporal progression of adult neurogenesis in the mouse SVZ, and that SOX9 is a target of miR-124 at the transition from transit-amplifying cell to neuroblast stage in the adult mouse brain $(23,24)$.

The present study aimed to investigate the association between the SOX9 gene and HSCR, by exploring the SOX9 and miR-124 expression patterns in patients with HSCR. To the best of our knowledge, the present study is the first investigate SOX9 and miR-124 detection in patients with HSCR, and may provide a novel understanding of HSCR for genetic counseling. 


\section{Materials and methods}

Patients. This study was approved by the Ethics Committee of China Medical University (Shenyang, China; 2012PS17K) and all subjects involved in the study provided written informed consent. Stenotic and normal colon segment tissues were obtained from 50 patients diagnosed with HSCR at the Department of Pediatric Surgery, Shengjing Hospital of China Medical University. Patient age ranged from 6 months to 6 years, with a mean age of 2.5 years. HSCR was diagnosed in patients using barium enema, acetylcholinesterase histochemical examination of the rectal mucosa and anorectal manometry and was further confirmed by the pathological results. At the time of surgery, none of these cases had received preoperative treatment, or had either a history of HSCR-associated or active enterocolitis. All tissue samples were subdivided for fixation using $4 \%$ paraformaldehyde solution, and for histological sectioning and storage at $-80^{\circ} \mathrm{C}$ for molecular analysis.

Antibodies. Polyclonal rabbit anti-SOX9 antibodies were purchased from Beijing Biosynthesis Biotechnology Co., Ltd. (no. bs-4177R; Beijing, China).

RNA extraction, reverse transcription and quantitative polymerase chain reaction $(q P C R$ ). A total of $\sim 100 \mathrm{mg}$ stenotic and normal intestine tissue from patients with HSCR were used for total RNA extraction using TRIzol ${ }^{\circledR}$ reagent (Invitrogen Life Technologies, Carlsbad, CA, USA), in accordance with the manufacturer's instructions. The harvested RNA was diluted to a concentration of $1 \mu \mathrm{g} / \mu \mathrm{l}$, aliquoted and stored at $-80^{\circ} \mathrm{C}$. For cDNA synthesis, two reagent kits were used: One Step PrimeScript $^{\circledR}$ miRNA cDNA Synthesis Kit and PrimeScript ${ }^{\mathbb{B}}$ RT reagent Kit (TaKaRa Biotechnology Co., Dalian, China). qPCR was performed in triplicate for each specimen using SYBR $^{\circledR}$ Green PCR Master Mix (TaKaRa Biotechnology Co.) in a LightCycler ${ }^{\circledR}$ (Roche Molecular Biochemicals, Co., Mannheim, Germany). RNA and miRNA expression levels were normalized to GAPDH mRNA and U6 miRNA expression levels, respectively. The primers (TaKaRa Biotechnology Co.) used in qPCR are listed in Table I. Following amplification, melting curve analysis was performed, using temperatures ranging from 60 to $90^{\circ} \mathrm{C}$, increasing by $0.2^{\circ} \mathrm{C}$ every $10 \mathrm{sec}$. The threshold cycle (CT) value, the point at which a significant increase in PCR product is detected, was also recorded. $\triangle \mathrm{CT}$ was calculated as follows: $\Delta \mathrm{CT}=\mathrm{CT}$ of gene of interest minus $\mathrm{CT}$ of GAPDH and U6. For these genes, one cycle change in CT corresponded to a $2.1 \pm 0.2$ standard error of the mean change in RNA dilution. To estimate the magnitude of the difference in expression for the other individual RNAs, the $\Delta \Delta \mathrm{CT}(\Delta \Delta \mathrm{CT}=\Delta \mathrm{CT}$ for samples with HSCR- $\Delta \mathrm{CT}$ for control samples) was transformed to 'fold change' $=2^{-\Delta \Delta c t}$.

Western blot analysis. Each specimen $(\sim 100 \mathrm{mg})$ of stenotic and normal intestine tissue from patients with HSCR was homogenized using surgical blades and sonicated in protein lysis buffer. Protein concentrations were then measured using the Bradford assay and specimens were adjusted to equal protein concentrations, aliquoted and stored at $-80^{\circ} \mathrm{C}$. Equal quantities of total proteins were separated by SDS-PAGE
Table I. Primer sequences for qPCR.

\begin{tabular}{llc}
\hline Target & \multicolumn{1}{c}{ Sequence $\left(5^{\prime}-3^{\prime}\right)$} & $\begin{array}{c}\text { Annealing } \\
\text { temperature } \\
\left({ }^{\circ} \mathrm{C}\right)\end{array}$ \\
\hline SOX9 & $\begin{array}{l}\text { F: GCAGCGAAATCAACGAG } \\
\text { R: CAAAGTCCAAACAGGCAGA }\end{array}$ & 51 \\
GAPDH & $\begin{array}{l}\text { F: AGAGCTACGAGCTGCCTGAC } \\
\text { R: AGC ACTGTGTTGGCGTACAGA }\end{array}$ & 55 \\
miR-124 & $\begin{array}{l}\text { F: CTCTCTCTCCGTGTTCACAG } \\
\text { R: GCTGTC AACGATACGCTACGTAACG }\end{array}$ & 53 \\
U6 & $\begin{array}{l}\text { F: CTCGCTTCGGCAGCACA } \\
\text { R: AACGCTTCACGAATTTGCGT }\end{array}$ & 60 \\
\hline
\end{tabular}

qPCR, quantitative polymerase chain reaction; SOX9, SRY-related HMG-box 9; miR-124, microRNA-124.

prior to being electro-transferred to polyvinylidene difluoride membranes (Millipore, Billerica, MA, USA). Membranes were then incubated with an anti-SOX9 polyclonal primary antibody (Beijing Biosynthesis Biotechnology Co. Ltd.) at a dilution of 1:50, overnight at $4^{\circ} \mathrm{C}$. Membranes were then washed, prior to incubation with a horseradish peroxidase-linked secondary antibody (Beijing Biosynthesis Biotechnology Co. Ltd) at a dilution of 1:2,000 for $1 \mathrm{~h}$ at room temperature. Blots were developed using an enhanced chemiluminescence kit and dectection was performed using a Syngene G:Box (SYOR4/1246; GE Healthcare, Pittsburgh, PA, USA).

Hematoxylin and eosin $(H \& E)$ staining and immunohistochemical staining. Immunohistochemical staining was performed as described previously (25). The stenotic and normal colon segments from patients with HSCR were immediately washed in cold phosphate-buffered saline (PBS; $\mathrm{pH}$ 7.4), prior to undergoing fixation in $4 \%$ buffered paraformaldehyde at $4^{\circ} \mathrm{C}$ for $24 \mathrm{~h}$. Subsequently, the samples were dehydrated, embedded in paraffin and sectioned sagittally at a thickness of $4 \mu \mathrm{m}$. Slides were then incubated in boiling $0.01 \mathrm{~mol} / \mathrm{l}$ citrate buffer ( $\mathrm{pH}$ 6.0) for $10 \mathrm{~min}$, cooled to room temperature and incubated with $3 \% \mathrm{H}_{2} \mathrm{O}_{2}$ for $15 \mathrm{~min}$, prior to being incubated with $10 \%$ normal goat serum (Biosynthesis Biotechnology Co., Ltd.) for $30 \mathrm{~min}$. Sections were then incubated with primary anti-SOX9 antibody, at a dilution of $1: 200$, at $4^{\circ} \mathrm{C}$ for $14-16 \mathrm{~h}$. Following primary antibody incubation, slides were washed in PBS, incubated with anti-rabbit immunoglobulin G-peroxidase antibody for $20 \mathrm{~min}$ at room temperature and then stained with 3'3-diaminobenzidine tetrahydrochloride (DAB). Dark brown granules in the cytoplasm and cytomembrane were considered positive results. Negative controls were generated by incubating slides with equivalent concentrations of nonimmune rabbit antiserum. Using integrated optical density (IOD) measurements (26), images were captured on color-positive films under identical illumination settings for each sample. The optical density (OD) of the cells was measured in the control tissues in the same fields, using a NIS-Elements Basic Research analysis system version 2.30 (Nikon Co., Kawasaki, Japan). The 


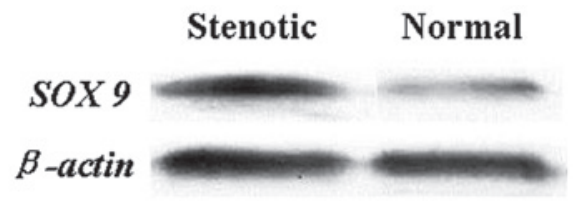

Figure 1. SOX9 expression in patients with HSCR. Protein extracts from normal and stenotic colon segment tissues from patients with HSCR were probed using western blot analysis with anti-SOX9 antibodies. HSCR, Hirschsprung's disease; SOX9, SRY-related HMG-box 9.

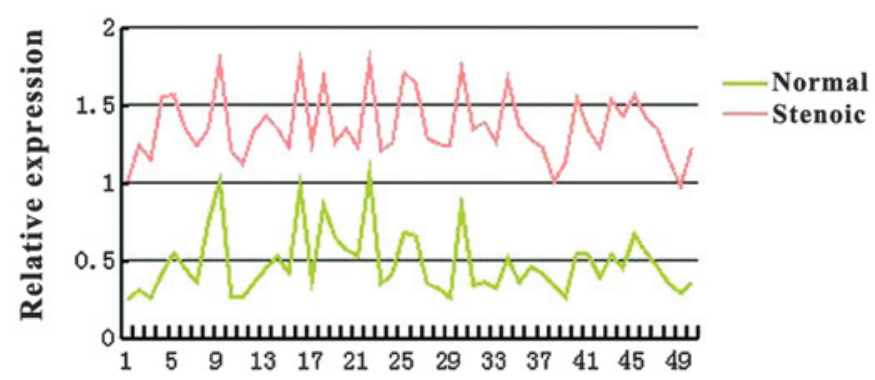

Figure 2. SOX9 protein expression relative to $\beta$-actin expression (y-axis) in normal and stenotic colon segments from patients with HSCR (x-axis). SOX9, SRY-related HMG-box 9; HSCR, Hirschsprung's disease.

A

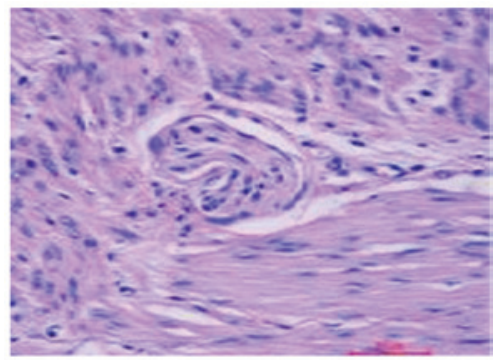

B

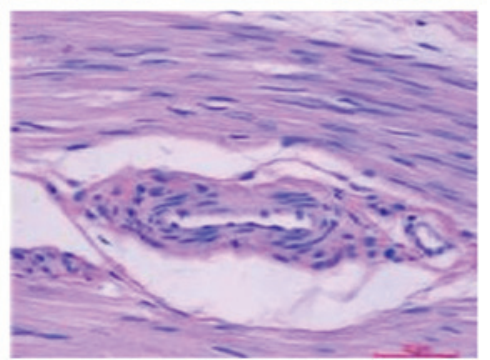

Figure 3. Photomicrographs of hematoxylin and eosin-stained stenotic and normal intestine tissue from patients with Hirschsprung's disease. (A) Stenotic colon segment tissue, showing aganglionosis (magnification, x400). (B) Normal colon segment tissue, showing the presence of ganglion cells (magnification, $\mathrm{x} 400$ ).

immunohistochemical-stained slides were independently reviewed by two researchers.

Statistical analysis. Statistical analyses were conducted using SPSS statistical software 16.0 (SPSS Inc., Chicago, IL, USA). $\mathrm{T}$ tests were used to determine the statistical significance of the differences in levels of miR-124 and SOX9 expression in stenotic colon segments compared with normal colon segments. All results are presented as the mean \pm standard deviation. A value of $\mathrm{P}<0.05$ was considered to indicate a statistically significant difference.
A

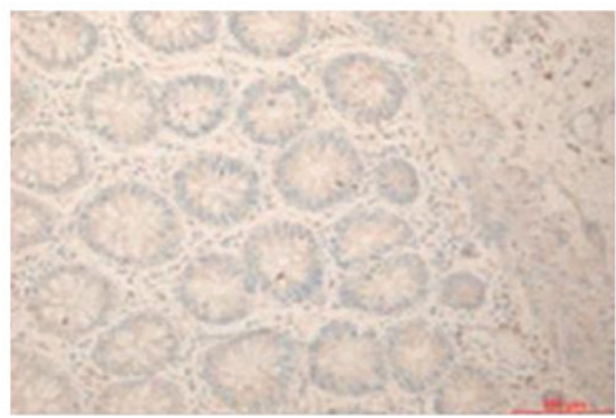

B

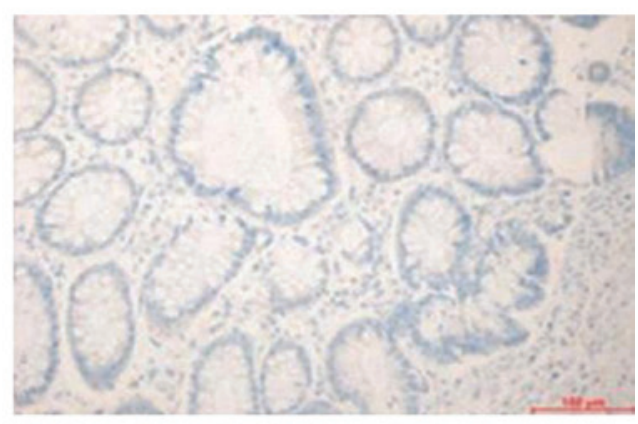

C

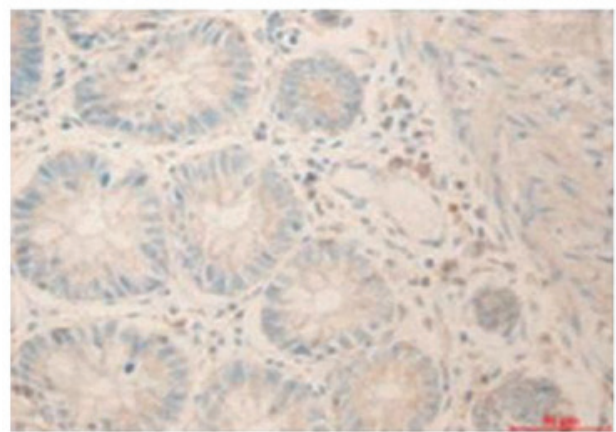

D

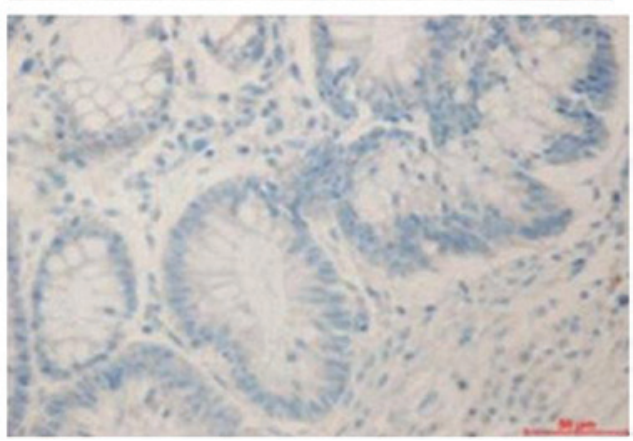

Figure 4. Distribution and expression of S0X9 using immunohistochemistry in patients with Hirschsprung's disease. (A and C) S0X9 staining in the mucous and muscular layers of stenotic colon segment tissues (magnifications, $x 200$ and $\times 400$, respectively). (B and D) S0X9 staining in the mucous and muscular layers of normal colon segment tissues (magnifications, x200 and x400, respectively). SOX9, SRY-related HMG-box 9.

\section{Results}

qPCR analysis. The concentration and purity of extracted total RNA of matched samples from 50 patients with HSCR was determined as the A260/280 ratio using a NanoVue ${ }^{\mathrm{TM}}$ spectrophotometer (GE Healthcare Bio-Sciences AB, Freiburg, Germany). The levels of SOX9 expression were normalized to the mRNA levels of GAPDH from the same specimen, and the levels of miR-124 expression were normalized to the levels of U6 miRNA expression. Melting curves for SOX9 and miR-124 were observed to differ between samples; 
therefore, for each sample, the replicates should share the same melting curve protocol for SOX9 and miR-124 amplification in the human transcriptome. It was observed that in patients with HSCR, the levels of SOX9 mRNA and miR-124 were 4.1 and 5.9 fold higher in the stenotic colon segment tissue than in the normal colon segment tissue, respectively $(\mathrm{P}<0.0047 ; \mathrm{P}<0.0007)$ (data not shown).

Protein expression levels. The protein expression of SOX9 was evaluated in the same 50 patients with HSCR using western blot analysis with specific antibodies. Consistent with the qPCR data, a significant increase in SOX9 was detected in the stenotic colon segment tissue compared with the matched normal colon segment tissue (Figs. 1 and 2). Densitometry analysis indicated that this difference was statistically significant $(\mathrm{P}<0.05)$.

Immunohistochemistry. The expression profile of SOX9 was further confirmed using immunohistochemistry. The stenotic colon segments were defined by the loss of the focal colon ganglion cells using H\&E staining (Fig. 3). Immunohistochemical staining of SOX9 revealed an increase in SOX9 expression in the stenotic colon segment tissue, as represented by the brown yellow staining (Fig. 4A and C). By contrast, SOX9 expression was observed to be lower in the normal colon segment tissue, where staining was light yellow or colorless (Fig. 4B and D).

\section{Discussion}

HSCR is the most common congenital gut motility disorder and is caused by the absence of ganglion cells in the bowel wall. It is well established that the onset of HSCR involves a series of complex processes, including the distortion of ganglion cell development at different stages $(3,26)$. Although progress has been made with regard to identifying some of the genetic factors associated with HSCR, numerous factors are yet to be elucidated. miRNA research is providing a novel understanding of the molecular mechanisms underlying normal and abnormal biological processes.

miRNAs are non-coding RNAs, 20-24 nucleotides in length, which are found in the majority of eukaryotic cells (27). Due to the complexity of HSCR, progress in understanding the disease is limited. miRNAs were originally identified as moderate biological modifiers of gene expression and protein translation; however, they have recently been identified as powerful regulators of diverse cellular processes, with significant roles in disease and tissue remodeling. Therefore, it has been suggested that miRNAs may represent target molecules for disease treatment (28). However, identifying the target genes regulated by miRNAs remains a challenge. It has been suggested that miRNAs and their target genes have a one-to-numerous and numerous-to-one interrelation in the function of miRNA regulation (26).

miR-124 is preferentially expressed in neurons and has been implicated in the positive modulation of the transitory progression of adult SVZ neurogenesis, through the repression of SOX9 (23). This suggests that miR-124 may be critical for the homeostasis of differentiation and proliferation in adult neural progenitor cells $(23,29)$. However, the miRNAs and specific target genes that are involved in the development of the ENS are yet to be identified. To the best of our knowledge, the present study was the first to detect miR-124 and SOX9 expression in patients with HSCR.

In the present study, the stenotic and normal colon segment tissues of 50 patients with HSCR were analyzed. The expression of SOX9 and miR-124 in stenotic and normal colon segments was detected using qPCR. The expression of SOX9 and miR-124 was observed to be significantly higher in stenotic colon segments than in normal colon segments $(\mathrm{P}<0.05)$. Furthermore, the results of the western blot analysis and immunohistochemical staining also revealed a significant increase in SOX9 expression in the stenotic colon segments compared with the normal colon segments.

miRNAs have recently attracted much attention as a novel research tool. It was observed in the present study that the miRNA that targets SOX9, miR-124, was upregulated in stenotic colon segments compared with the normal colon segments. Therefore, this study concludes that an association may exist between the expression of SOX9 and miR-124 in patients with HSCR. miR-124 may be capable of promoting the development of HSCR by targeting SOX9; however, the mechanism by which this occurs is yet to be elucidated.

In conclusion, the present study reveals that the expression of SOX9 is abnormal in patients with HSCR, and that miR-124 may be a risk factor for these patients. Furthermore, it has been demonstrated that SOX 9 may represent a potential target for gene therapy and may be a promising candidate biomarker for HSCR. Further investigations are required to assess the potential of SOX9 for the treatment and diagnosis of HSCR and to identify other miRNAs that may be involved in HSCR.

\section{Acknowledgments}

The present study was supported by a grant from the National Natural Science Foundation of China (no. 30772277).

\section{References}

1. Amiel $\mathbf{J}$ and Lyonnet S: Hirschsprung disease, associated syndromes, and genetics: a review, J Med Genet 38: 729-739, 2001.

2. Whitehouse FR and Kernohan JW: Myenteric plexus in congenital megacolon; study of 11 cases. Arch Intern Med (Chic) 82: 75-111, 1948.

3. Haricharan RN and Georgeson KE: Hirschsprung disease. Semin Pediatr Surg 17: 266-275, 2008.

4. Tam PK and Garcia-Barceló M: Genetic basis of Hirschsprung's disease. Pediatr Surg Int 25: 543-558, 2009.

5. Angrist M, Kauffman E, Slaugenhaupt SA, et al: A gene for Hirschsprung disease (megacolon) in the pericentromeric region of human chromosome 10. Nat Genet 4: 351-356, 1993.

6. Luo Y, Ceccherini I, Pasini B, et al: Close linkage with the RET protooncogene and boundaries of deletion mutations in autosomal dominant Hirschsprung disease. Hum Mol Genet 2: 1803-1808, 1993.

7. Lyonnet S, Bolino A, Pelet A, et al: A gene for Hirschsprung disease maps to the proximal long arm of chromosome 10 . Nat Genet 4: 346-350, 1993.

8. Amiel J, Attié T, Jan D, et al: Heterozygous endothelin receptor $\mathrm{B}(\mathrm{EDNRB})$ mutations in isolated Hirschsprung disease. Hum Mol Genet 5: 355-357, 1996.

9. Attié T, Till M, Pelet A, Amiel J, Edery P, Boutrand L, Munnich A and Lyonnet S: Mutation of the endothelin-receptor B gene in Waardenburg-Hirschsprung disease. Hum Mol Genet 4: 2407-2409, 1995. 
10. Kusafuka T, Wang Y and Puri P: Novel mutations of the endothelin-B receptor gene in isolated patients with Hirschsprung's disease. Hum Mol Genet 5: 347-349, 1996.

11. Edery P, Attié T, Amiel J, Pelet A, Eng C, Hofstra RM, Martelli H, Bidaud C, Munnich A and Lyonnet S: Mutation of the endothelin-3 gene in the Waardenburg-Hirschsprung disease (Shah-Waardenburg syndrome). Nat Genet 12: 442-444, 1996.

12. Hofstra RM, Osinga J, Tan-Sindhunata G, et al: A homozygous mutation in the endothelin-3 gene associated with a combined Waardenburg type 2 and Hirschsprung phenotype (Shah-Waardenburg syndrome). Nat Genet 12: 445-447, 1996.

13. Angrist M, Bolk S, Halushka M, Lapchak PA and Chakravarti A Germline mutations in glial cell line-derived neurotrophic factor (GDNF) and RET in a Hirschsprung disease patient. Nat Genet 14: 341-344, 1996.

14. Ivanchuk SM, Myers SM, Eng C and Mulligan LM: De novo mutation of GDNF, ligand for the RET/GDNFR-alpha receptor complex, in Hirschsprung disease. Hum Mol Genet 5: 2023-2026, 1996.

15. Salomon R, Attié T, Pelet A, et al: Germline mutations of the RET ligand GDNF are not sufficient to cause Hirschsprung disease. Nat Genet 14: 345-347, 1996.

16. Pingault V, Bondurand N, Kuhlbrodt K, et al: SOX10 mutations in patients with Waardenburg-Hirschsprung disease. Nat Genet 18 171-173, 1998.

17. Southard-Smith EM, Angrist M, Ellison JS, Agarwala R, Baxevanis AD, Chakravarti A and Pavan WJ: The Sox10(Dom) mouse: modeling the genetic variation of Waardenburg-Shah (WS4) syndrome. Genome Res 9: 215-225, 1999.

18. Sham MH, Lui VC, Fu M, Chen B and Tam PK: SOX10 is abnormally expressed in aganglionic bowel of Hirschsprung's disease infants. Gut 49: 220-226, 2001.
19. Stolt CC, Lommes P, Sock E, Chaboissier MC, Schedl A and Wegner M: The Sox 9 transcription factor determines glial fate choice in the developing spinal cord. Genes Dev 17: 1677-1689, 2003.

20. Kordes U, Cheng YC and Scotting PJ: Sox group E gene expression distinguishes different types and maturational stages of glial cells in developing chick and mouse. Brain Res Dev Brain Res 157: 209-213, 2005

21. Ambros V: The functions of animal microRNAs. Nature 431: $350-355,2004$.

22. Kloosterman WP and Plasterk RH: The diverse functions of microRNAs in animal development and disease. Dev Cell 11: 441-450, 2006.

23. Cheng LC, Pastrana E, Tavazoie M and Doetsch F: miR-124 regulates adult neurogenesis in the subventricular zone stem cell niche. Nat Neurosci 12: 399-408, 2009.

24. Grandjean V, Gounon P, Wagner N, Martin L, Wagner KD, Bernex F, Cuzin F and Rassoulzadegan M: The miR-124-Sox9 paramutation: RNA-mediated epigenetic control of embryonic and adult growth. Development 136: 3647-3655, 2009

25. Simic P and Vukicevic S: Bone morphogenetic proteins in development and homeostasis of kidney. Cytokine Growth Factor Rev 6: 299-308, 2005

26. Martucciello G: Hirschsprung's disease, one of the most difficult diagnoses in pediatric surgery: a review of the problems from clinical practice to the bench. Eur J Pediatr Surg 18: 140-149, 2008.

27. Bartel DP: MicroRNAs: genomics, biogenesis, mechanism, and function. Cell 116: 281-297, 2004.

28. van Rooij E: The art of microRNA research. Circ Res 108 219-234, 2011

29. Papagiannakopoulos T and Kosik KS: MicroRNA-124: micromanager of neurogenesis. Cell Stem Cell 4: 375-376, 2009. 